Int. J. Electrochem. Sci., 14 (2019) 7258 - 7269

\title{
One-pot preparation of three-dimensional macroporous phosphomolybdic acid-MoS 2 -reduced graphene oxide hybrid for electrochemical detection of nitrite
}

\author{
Hui Xu ${ }^{l}$, Guangran Ma ${ }^{1,2}$, Meijuan $W u^{l}$, Xia Peng ${ }^{l}$, Lin Wang ${ }^{l}$, Fugang $X u^{l, *}$ \\ ${ }^{1}$ College of Chemistry and Chemical Engineering, Jiangxi Normal University, Nanchang 330022, \\ China \\ ${ }^{2}$ Analytical and Testing Center of Jiangxi Normal University, Nanchang, 330022, China \\ *E-mail: fgxu@jxnu.edu.cn
}

doi: $10.20964 / 2019.08 .81$

Received: 12 April 2019 / Accepted: 5 June 2019 / Published: 30 June 2019

In this study, a new hybrid PMA-MoS 2 -rGO composed of phosphomolybdic acid (PMA), $\mathrm{MoS}_{2}$, and reduced graphene oxide ( $\mathrm{rGO}$ ) was prepared for sensitive electrochemical detection of nitrite. The hybrid was prepared by a facile one-pot hydrothermal reaction, and PMA was introduced as active species for catalytic oxidation of nitrite and Mo source of $\mathrm{MoS}_{2}$. The structure and component of the product were characterized by SEM, Raman and EDS, and its catalysis toward nitrite oxidation was investigated by several electrochemical methods. The results revealed the hybrid composed of flower-sphere-like $\mathrm{MoS}_{2}$ interwind with rGO sheet while PMA doped in them, forming a three-dimensional porous structure. The $\mathrm{MoS}_{2}$ flower-sphere greatly reduced the aggregation of rGO and increased active surface area of the hybrid. Introducing rGO improved the conductivity and greatly enhanced the catalytic current. PMA played a dominate role in catalytic oxidation of nitrite. Owning to these synergy interactions, the hybrid exhibited greatly enhanced catalysis for nitrite oxidation than $\mathrm{MoS}_{2}$ or PMA-MoS 2 . The hybrid was used for fabricating a nitrite electrochemical sensor, which showed a linear rang of $0.5 \mu \mathrm{M}$ to $8000 \mu \mathrm{M}$ and a LOD of $0.2 \mu \mathrm{M}(\mathrm{S} / \mathrm{N}=3)$. The sensor also showed good selectivity and stability with satisfactory recoveries for real sample test. The PMA-MoS 2 -rGO hybrid with easy preparation, porous structure, high stability and good catalysis holds great potential in environmental monitoring, chemical analysis, and so on.

Keywords: $\mathrm{MoS}_{2}$, polyoxometalates, nitrite, graphene, nanohybrid, electrocatalysis.

\section{$\underline{\text { FULL TEXT }}$}

(C) 2019 The Authors. Published by ESG (www.electrochemsci.org). This article is an open access article distributed under the terms and conditions of the Creative Commons Attribution license (http://creativecommons.org/licenses/by/4.0/). 